\title{
28 Research Square \\ Lung Adenocarcinoma Isolated Metastasizing to Pancreas a Case Report and Review
}

\section{Ludong Tan ( $\nabla$ tld@jlu.edu.cn )}

Jilin University First Hospital

Yu Fu

Jilin University First Hospital

Shupeg Wang

Jilin University First Hospital

Jiang Peiqiang

Jilin University First Hospital

Yahui Liu

Jilin University First Hospital https://orcid.org/0000-0003-3397-2714

\section{Research Article}

Keywords: lung adenocarcinoma, pancreatic secondary tumor, surgical treatment.

Posted Date: October 13th, 2021

DOl: https://doi.org/10.21203/rs.3.rs-69263/v2

License: (c) (1) This work is licensed under a Creative Commons Attribution 4.0 International License. Read Full License 


\section{Abstract}

BACKGROUND: Lung cancers are prone to metastasis. It is commonly associated with metastases in the brain, bone, and liver.However, isolated pancreatic metastasis are extremely rare in lung adenocarcinoma patients $\square$

CASE SUMMARY: A Chinese man underwent pulmonary lobectomy for adenocarcinrcinoma 6 years ago. He was referred to our department because that postoperative reexamination(Abdomen CT) revealed space occupying lesions in the pancreatic neck,and no abnormality was found in any other organ. Therefore, it was considered to pancreatic carcinoma. Pancreatectomy of the pancreatic body and tail, as well as splenectomy, were performed,and he was given four courses of Pemetrexed and Carboplatin chemotherapy after one month of operation. Then the gefitinib was given.A year after surgery, the patient survived without tumor.

CONCLUSION: Secondary pancreatic tumors are rare in clinical practice.It is the longer tumor-free survival in patients with isolated pancreatic metastasis of lung adenocarcinoma.However,the value of surgical treatment for isolated metastasis of lung adenocarcinoma needs further discussion.

\section{Background}

Lung cancers have the highest morbidity and mortality among malignant diseases in the world; and adenocarcinoma of the lung, which is a common type of lung cancer, has particularly poor sensitivity to radiotherapy and chemotherapy ${ }^{[1]}$.Lung cancers are commonly associated with metastases in the brain, bone, and liver, and other organs. Pancreatic metastasis in lung cancer patients, however, is very rare, with published probabilities in the range of $6-11 \%$ [2]. Pancreatic metastases, which are often found in patients with metastatic clear cell carcinoma of the kidney, colorectal cancer, and sarcomas, are rarely isolated, but rather are usually accompanied by metastasis of other organs ${ }^{[3.4]}$.The probability of solitary pancreatic-occupying lung cancer metastasis has been reported to be only $0.3-3.0 \%[305,06]$.

Here, we report the case of a man who underwent pulmonary lobectomy for adenocarcinoma of the lung and then resection of apparent metastases occupying the pancreatic neck and body 6 years later. A brief review of the relevant literature follows.

\section{Case Presentation}

Six years ago, a 56-year-old Chinese male patient was admitted to the First Hospital of Jilin University for a pulmonary space-occupying lesion revealed by computerized tomography(CT). No obvious abnormalities were apparent in a physical examination and the patient did not have cough, expectoration, hemoptysis, or any other apparent discomfort. The patient had no family history of respiratory or digestive tract diseases. He was not a smoker or alcohol abuser. Liver function tests at the time of admission showed the following: aspartate aminotransferase, $29 \mathrm{U} / \mathrm{L}$; alanine aminotransferase, $32 \mathrm{U} / \mathrm{L}$, 
alkaline phosphatase, 12,348 U/L, total protein, $76.4 \mathrm{~g} / \mathrm{L}$; albumin, $46.6 \mathrm{~g} / \mathrm{L}$; total bilirubin; $16.5 \mu \mathrm{mol} / \mathrm{L}$; direct bilirubin, $4.0 \mu \mathrm{mol} / \mathrm{L}$. Meanwhile, we obtained the following routine blood test results: leukocytes, $5.72 \times 10^{9} / \mathrm{L}$, red blood cells $5.43 \times 10^{12} / \mathrm{L}$, hemoglobin: $166 \mathrm{~g} / \mathrm{L}$, platelet: $253 \times 10^{9} / \mathrm{L}$. serological tumour marker:CEA:12.97ng/ml,CA125:13.14U/ml,CA199:17.55U/ml.

A chest computerized tomography(CT) with secondary enhancement demonstrated ground glass density nodule (approximate size, $2.1 \times 2.2 \mathrm{~cm}$ ) in the upper lobe of the left lung with irregular edges, burrs, lobes, and vacuoles. Imaging-based differential diagnosis led us to exclude alveolar carcinoma as vitreous nodules in the upper lobe of the left lung.

The patient underwent thoracoscopic upper lobe resection of the left lung. Postoperative Pathological Examination howed infiltrating adenocarcinoma with medium differentiation(Figure 2A). Most of the abnormal tissue ( 90\%) showed an acinar growth pattern, whereas some showed a micropapillary growth pattern $(10 \%)$. The tumor volume was about $2.0 \times 1.5 \times 1.2 \mathrm{~cm}$. No metastases were found in the visceral pleura, bronchiole walls, bronchotomy margins, vascular incision margins, vasculature, nerves, or para-bronchogenic lymph nodes. Tumor-node-metastasis staging was T1NOM0, with a pathological stage of $1 \mathrm{~A}$.

A regular scheduled follow-up re-examination 6 years after the lumg tumor resection in January of 2018 revealed untreated pancreatic neck lesions. A subsequent planned re-examination revealed that the mass in the pancreatic neck had increased in volume(Figure1). The patient was admitted to the First Hospital of Jilin University for secondary distal pancreatic atrophy and pancreatic duct dilation. Based on these imaging findings, we made a preoperative working diagnosis of space-occupying lesions of the pancreatic neck, secondary distal pancreatic contraction, and expansion of the pancreatic duct; primary pancreatic cancer could not be ruled out preoperatively. No metastases were observed in head and chest CT scans. The apparent mass were treated surgically.

Following distal pancreatectomy and splenectomy, the patient's postoperative recovery went well, with no complications (e.g. hemorrhage, pancreatic leakage, abdominal infection). In Postoperative Pathological Examination, the mass measured $2.3 \times 2.0 \times 1.8 \mathrm{~cm}$, was pale yellow color, and a firm, tough consistency.postoperative pathological findings showed adenocarcinoma infiltration in the pancreatic tissue, including Naspsin A+ (Figure 2B) and thyroid transcription factor 1+ (TTF-1) (Figure 2C) .By patient's medical history, these postoperative pathological findings were demonstrative of metastatic lung adenocarcinoma infiltration of pancreatic tissue(Figure 2D). Detection for gene of the mass revealed a deletion mutation in exon 19 of EGFR. Based on the above findings, we diagnosed the patient with isolated pancreatic metastasis of lung cancers $\square$

He was given four courses of pemetrexed and carboplatin chemotherapy after one month of operation . Then the gefitinib was given.A year after the operation,the patient underwent a full body examination.Abdominal CT images(Fig3A, 3B) Head(Fig3C) and chest(Fig3E), with and without 
enhancement, conducted showed no abnormalities.Meanwhile a systemic bone(Fig3D) scan also showed no abnormalities.

\section{Discussion And Conclusions}

Here, we reported the case of a lung cancer patient whose isolated Pancreatic metastasis could be resected surgically. Due to the rarity of lung metastases in the pancreas and the extreme rarity of isolated pancreatic metastases, there is limited representation of this clinical presentation and its treatment in the literature. Lee et al. ${ }^{[8]}$ reported that surgical resection of secondary pancreatic tumors can improve the postoperative survival rate of patients, depending on the tumor's pathological type, whether there has been vascular invasion, and the marginal state of the primary tumor. In a multi-center study, Moussa and colleagues showed that surgical treatment of pancreatic metastases of renal or colorectal primary cancers tumor can improve patient survival ${ }^{[9]}$. Similarly, Zerbi et al. ${ }^{[10]}$ reported that surgical treatment for pancreatic metastasis of renal cancer can prolong 5-year survival, and Aimoto et al. ${ }^{[11]}$ reported a case of a patient with isolated pancreatic metastasis of renal cancer with a successful surgical treatment. Because isolated pancreatic metastasis of lung cancer is rare, and isolated pancreatic metastasis of lung cancer in particular is extremely rare, there are few cases of surgical resection for isolated pancreatic metastasis of lung cancer, and the value of surgical resection has not been clarified. Igai and colleagues ${ }^{[12]}$ reported the case of a 67-year-old male patient who underwent pancreaticoduodenal resection of a metastasis from a lung cancer tumor that had been removed 5 years prior and remained free of recurrence at his 6-month postoperative follow-up. Watanabe et al. ${ }^{[13]}$ reported the case of 64-year-old male patient who underwent right pulmonary resection due to adenocarcinoma of the right lung and then, 1 year later, had a pancreatic tail mass resected. Mori et al. ${ }^{[14]}$ reported the case of a patient who underwent lobectomy for lung cancer, carboplatin-paclitaxel chemotherapy for 6 months, and subsequent successful surgical resection of an isolated pancreatic metastasis followed by postoperative chemotherapy, with no recurrence at the 6 month-follow-up examination. Despite these encouraging case reports, however, Reddy et al. reported a mean survival time of only 8.75 months for pancreatic secondary tumors, with a $0 \% 5$-year survival rate ${ }^{[7]}$. However, this Chinese male patient who with isolated pancreatic metastases of lung cancer underwent surgical treatment, but still no tumor recurrence was found six months later,so the value of surgical treatment for isolated metastasis of lung cancer needs further discussion. The indications for surgical treatment of metastatic pancreatic tumors are unclear. Wilson et al. ${ }^{[15]}$, who suggested that surgical removal of isolated pancreatic metastases may extend patient survival to some extent, urged clinicians to be attentive

to the following two considerations before pursuing surgical resection: 1) the type of primary disease; and 2) whether the isolated distant metastasis has occurred after a long disease-free period. Notwithstanding, it is very difficult to diagnose pancreatic metastases by preoperative imaging. Because pancreatic metastatic tumors and primary pancreatic tumors are similar in size, location, and number of tumors ${ }^{[16]}$, imaging alone cannot distinguish between them. Differential diagnosis between the two may require endoscopic ultrasonography-guided puncture biopsy ${ }^{[17]}$. 
In conclusion, this report has described a rare disease isolated pancreatic metastasis of lung cancers. It reminds us the possibility of isolated metastasis in the pancreas. Postoperative pathological examination should be mindful of the possibility of isolated pancreatic metastasis.Meanwhile, further studies are needed to clarify whether surgical treatment of isolated pancreatic metastases of lung cancer improve patient survival as has been shown for renal and colorectal cancer patients.

\section{Abbreviations}

$\mathrm{CT}$ = computerized tomography. EGFR=epidermal growth factor receptor.

\section{Declarations}

Ethics approval and consent to participate $\ T$ The study was approved by the The First Hospital Of Jilin University Institutional Review Board for human studies.

Consent for publication $邓$ Written informed consent was obtained from the patient for publication of this case report and accompanying images.

Availability of data and materials $₫$ All data generated or analyzed during this study are included in this published article.

Competing interests $₫$ The authors declare that they have no competing interests

Funding $\triangle$ We declare that we have not received any funding.

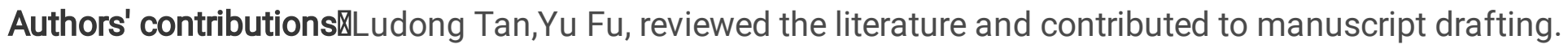

Shupeng Wang,Peiqiang Jiang,Yahui Liu, were responsible for the revision of the manuscript for important intellectual content.

Acknowledgements $\varangle$ We would like to thank Professor Liu for putting forward this idea and revising our article

\section{References}

[1] Siegel RL, Miller KD, Jemal A. Cancer statistics, 2016. CA Cancer J Clin, 2016, 66(1): 7-30.

[2] Adsay NV, Andea A, Basturk O, et al. Secondary tumors of the pancreas: an analysis of a surgical and autopsy database and review of the literature. Virchows Arch 2004; 444:527-35.

[3] Dar FS, Mukherjee S, Bhattacharya S. Surgery for secondary tumors of the pancreas. HPB (Oxford) 2008; 10:498-500. 
[4]Reddy S, Edil BH, Cameron JL,et al. Pancreatic resection of isolated metastases from nonpancreatic primary cancers. Ann Surg Oncol 2008; 15:3199-206.

[5] Sperti C, Pasquali C, Liessi G, et al. Pancreatic resection for metastatic tumors to the pancreas. J Surg Oncol 2003; 83:161-6.

[6]Bahra M, Jacob D, Langrehr JM, et al. Metastatic lesions to the pancreas. When is resection reasonable?. Chirurg 2008; 79:241-8.

[7] Reddy S, Wolfgang CL. The role of surgery in the management of isolated metastases to the pancreas. Lancet Oncol 2009; 10: 287-93

[8] Lee SR, Gemenetzis G, Cooper M, et al. Long-term outcomes of 98 surgically resected metastatic tumors in the pancreas. Ann Surg Oncol. 2017; 24(3):801-807.

[9] Moussa A, Mitry E, Hammel P, et al. Pancreatic metastases: a multicentric study of 22 patients. Gastroenterol Clin Biol 2004; 28:872-6.

[10] Zerbi, A., Ortolano, E., Balzano, G. et al. Pancreatic metastasis from Renal cell carcinoma: Which patients benefit from surgical resection? Ann Surg Oncol. 2008; 15:1161.

[11] Aimoto T, Uchida E, Yamahatsu K, et al. Surgical treatment for isolated multiple pancreatic metastases from renal cell carcinoma: report of a case. J Nippon Med Sch. 2008; 75(4):221-4.

[12] Igai $H$, Kamiyoshihara $M$, Nagashima $T$ et al. A resectable pancreatic metastasis from pulmonary adenocarcinoma. Ann Thorac Cardiovasc Surg. 2014;20(3):243-5. Epub 201

[13] Watanabe T, Morinaga S, Numata M. et al. Pancreatic resection for metastatic tumors to the pancreas .Gan to Kagaku Ryoho. 2011; 38(12):2068-70.

[14] Mori N, Sawada T, Satoh H,et al.A resected case of solitary pancreatic metastasis from adenocarcinoma of the lung. JOP. 2008; 9(6):698-703

[15] Wilson RL, Brown RK, Reisman D.Surgical resection for metastatic non-small cell lung to the pancreas.Lung Cancer. 2009; 63(3):433-5.

[16] DeWitt J, Jowell P, Leblanc J, et al. EUS-guided FNA of pancreatic metastases: A multicenter experience. Gastrointest Endosc. 2005; 61:689-96

[17] Alomari AK, Ustun B, Aslanian HR,et al. Endoscopic ultrasound-guided fine-needle aspiration diagnosis of secondary tumors involving the pancreas: An institution's experience. CytoJournal 2016; $13: 1$

\section{Figures}




\section{$1 \mathrm{~A}$}

\section{$1 \mathrm{~B}$}

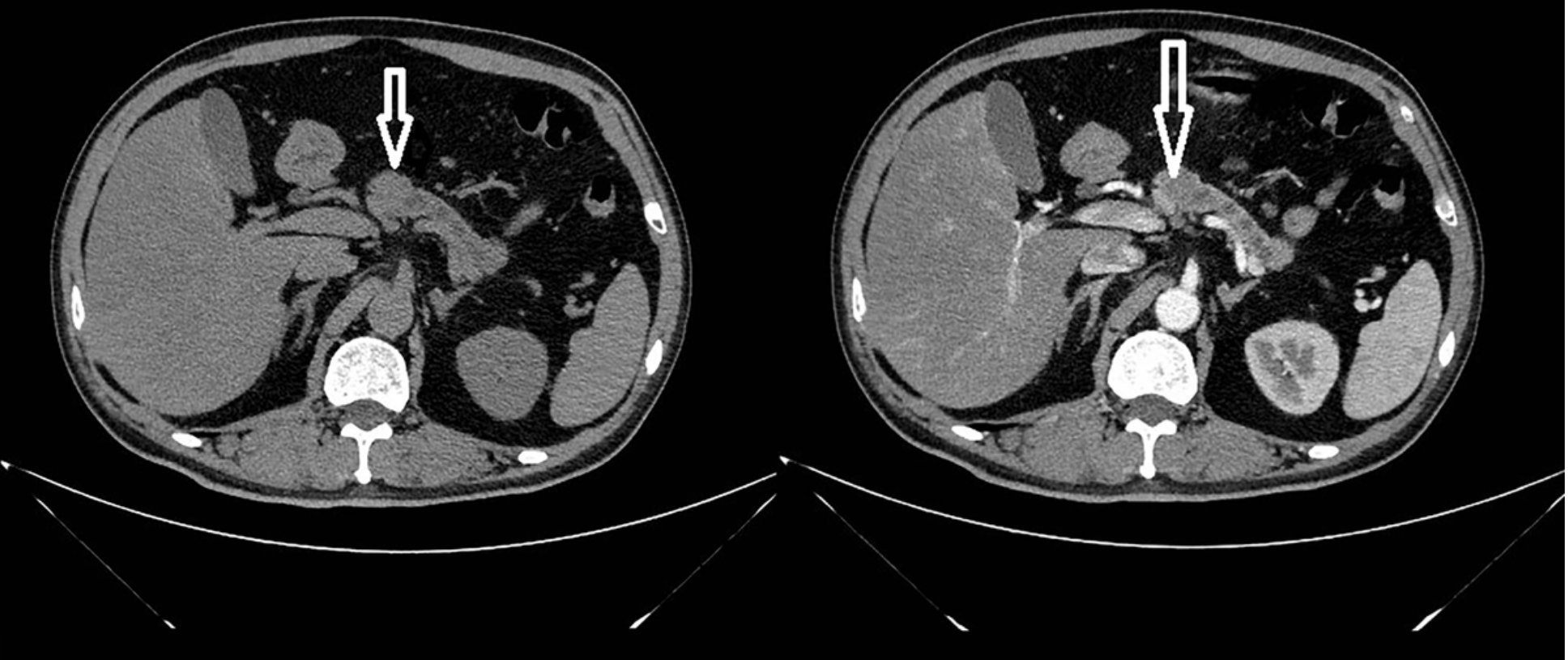

\section{$1 \mathrm{C}$}
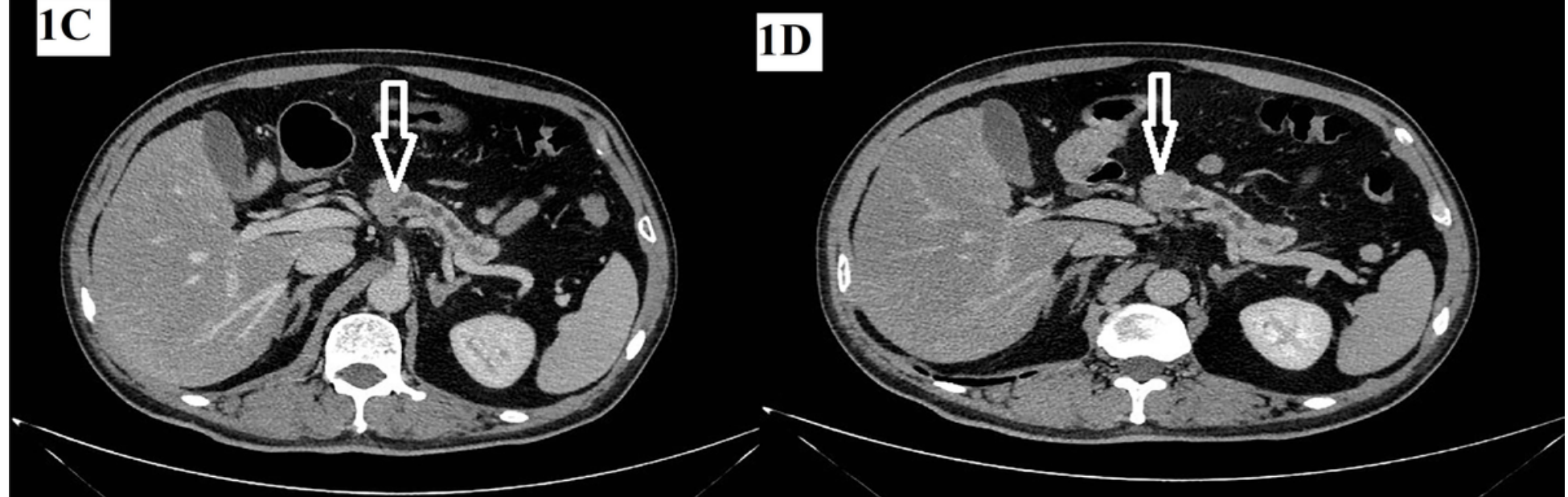

\section{Figure 1}

Enhanced axial abdominal CT image series showing a nodular low-density shadow in the pancreatic head and neck. Secondary distal pancreatic atrophy can be seen and dilatation of the pancreatic duct can be seen in panel. 


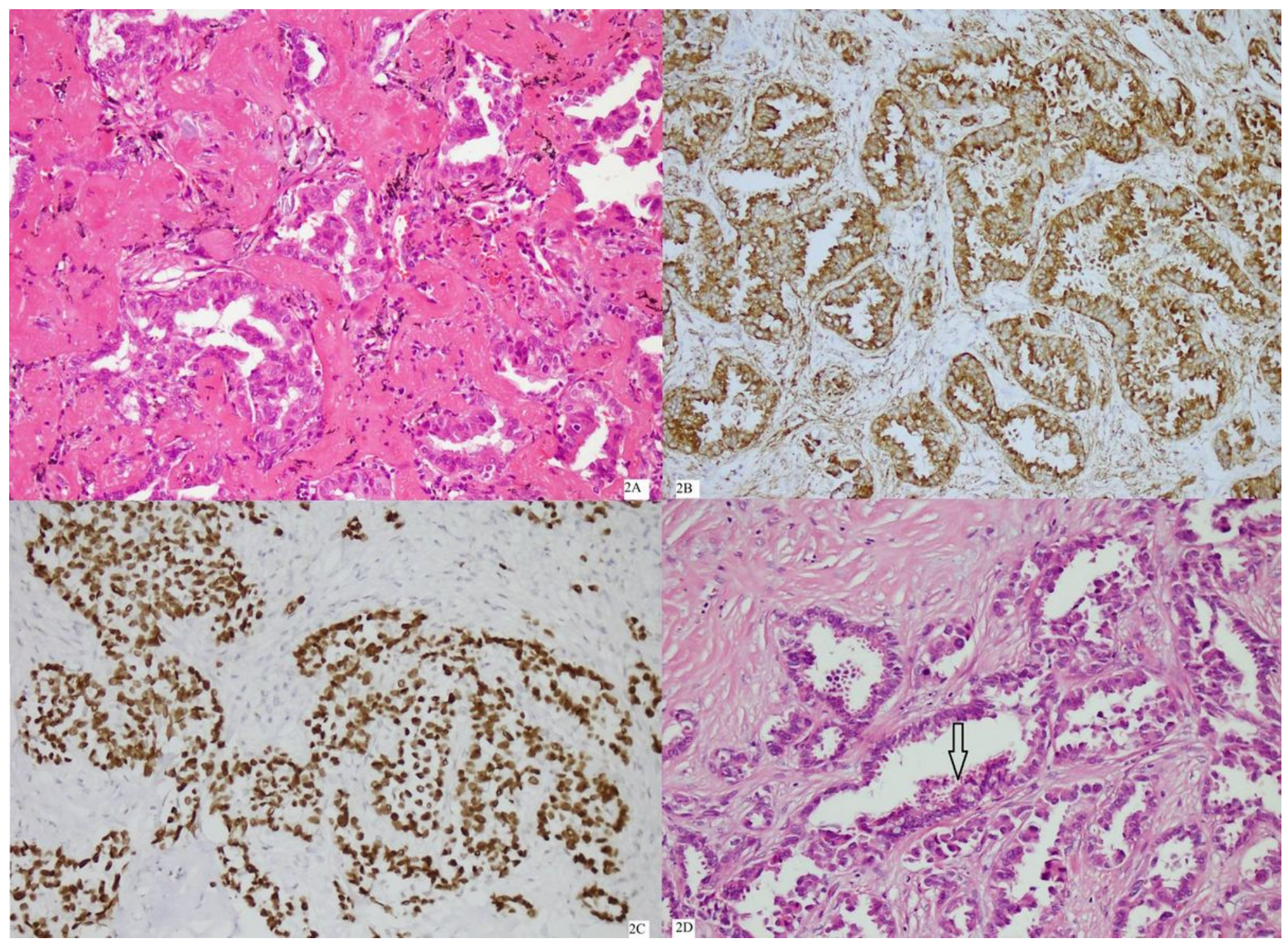

Figure 2

(1A) Histology and immunohistochemistry demonstrating lung infiltrating adenocarcinoma. (1B) H-E stained Napsin A of section near tissue pancreas was immunopositivity in panel. (1C) TTF-1 of section near tissue pancreas was immunopositivity.It showed the adenocarcinoma component. (1D) H-E stained section showed the arrow head indicated the component of adenocarcinoma) All photomicrographs, 200x. 


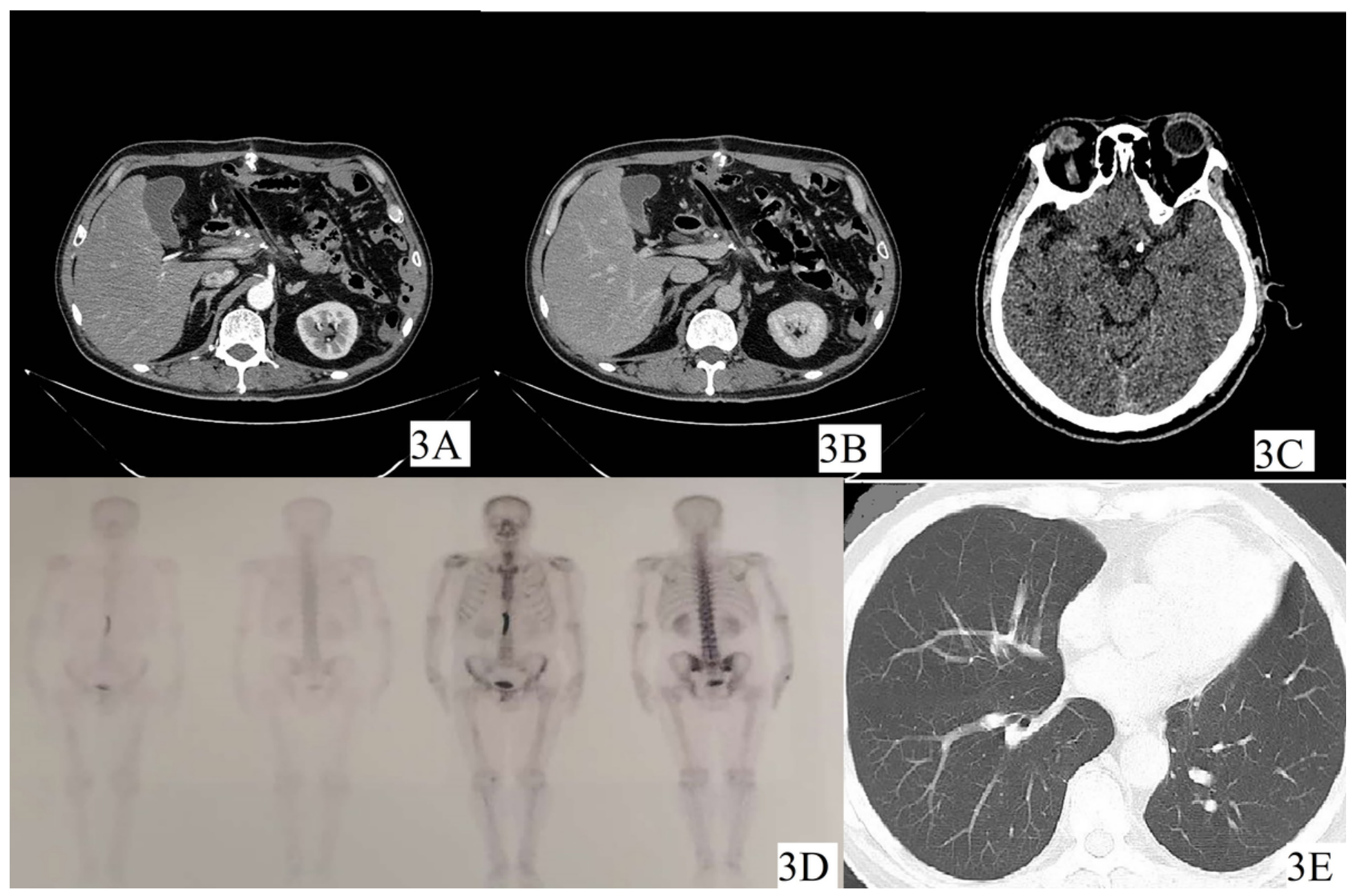

Figure 3

Abdominal CT (Fig3A, Fig3B), chest CT(Fig3D), head CT(Fig3C) and bone scanning (Fig3E) with no signs of metastasis. 